\title{
Supporting Information: Crystallinity and size control of colloidal germanium nanoparticles from organogermanium halide reagents.
}

Bruno Pescara, ${ }^{[1,2,3]}$ Katherine A. Mazzio,,${ }^{[1,2, *}$ Klaus Lips, ${ }^{[1,2,4]}$ Simone Raoux, ${ }^{[1,2,5]}$

${ }^{1}$ Institute for Nanospectroscopy, Helmholtz-Zentrum Berlin für Materialien und Energie GmbH, Albert-Einstein-Straße 15, 12489 Berlin, Germany

${ }^{2}$ Energy Materials In-Situ Laboratory Berlin (EMIL), Helmholtz-Zentrum Berlin für Materialien und Energie GmbH, AlbertEinstein-Straße 15, 12489 Berlin, Germany

${ }^{3}$ Department of Chemistry, Humboldt-Universität zu Berlin, Brook-Taylor-Straße 2, 12489 Berlin, Germany

${ }^{4}$ Department of Physics, Freie Universität Berlin, Arnimallee 14, 14195 Berlin, Germany

${ }^{5}$ Department of Physics, Humboldt-Universität zu Berlin, Newtonstraße 15, 12489 Berlin, Germany 


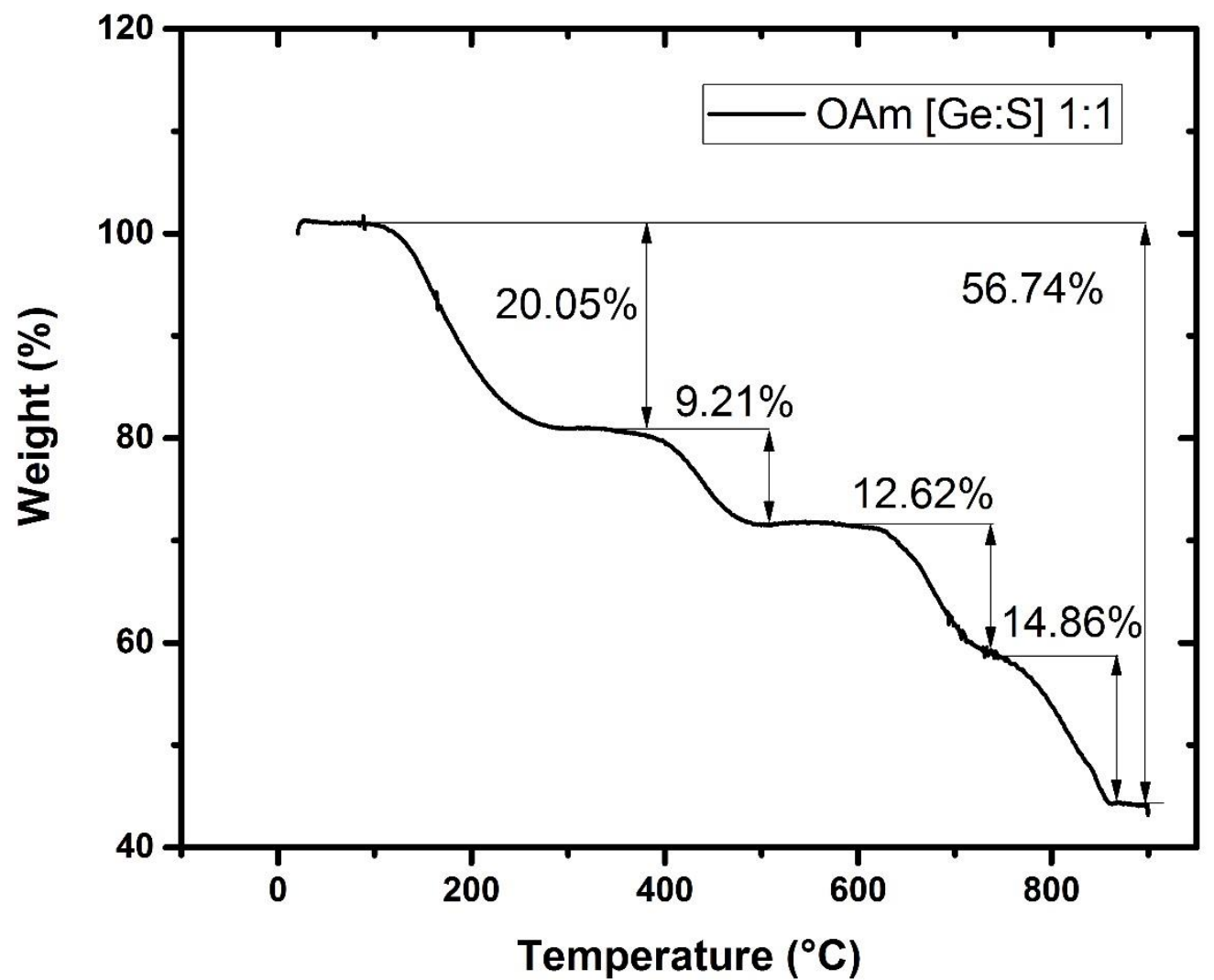

Figure S1: Example of Thermogravimetric analysis for a sample synthetized with OAm and [Ge:S] 1:1. The profile shows a first mass loss at low temperature associated with the evaporation of Sulfur plus residual solvent from the work up. At around $350{ }^{\circ} \mathrm{C}$, which corresponds to the boiling point of oleylamine, a second smaller loss arises due to the evaporation of the residual oleylamine. At higher temperatures, two additional mass losses are observed. The first at $550{ }^{\circ} \mathrm{C}$ is attributed to the detachment of unbound polymer residue, while the second corresponds to the detachment of the capping agents directly ligated to the NPs surface. The broad and almost overlapping nature of the last two losses is due to complex composition of the polymer resulting from the subsequent reactions shown in Scheme 1 reactions 3-6. 

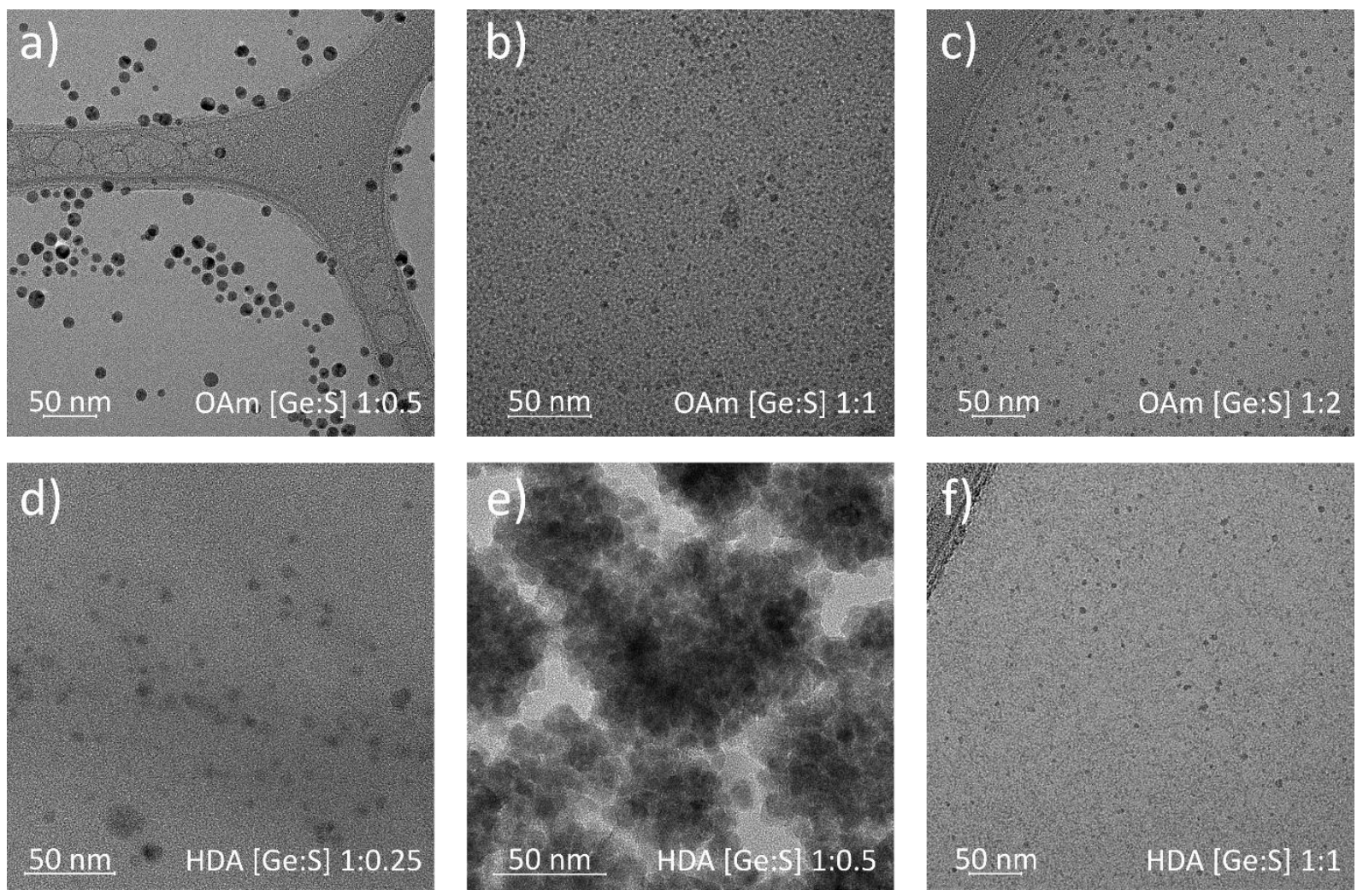

Figure S2: Low magnification TEM images for all samples synthesized with a) OAm [Ge:S] 1:0.5, b) OAm [Ge:S] 1:1, c) OAm [Ge:S] 1:2, d) HDA [Ge:S] 1:0.25, e) HDA [Ge:S] 1:0.5, and f) $\operatorname{HDA}[\mathrm{Ge}: \mathrm{S}] 1: 1$. 

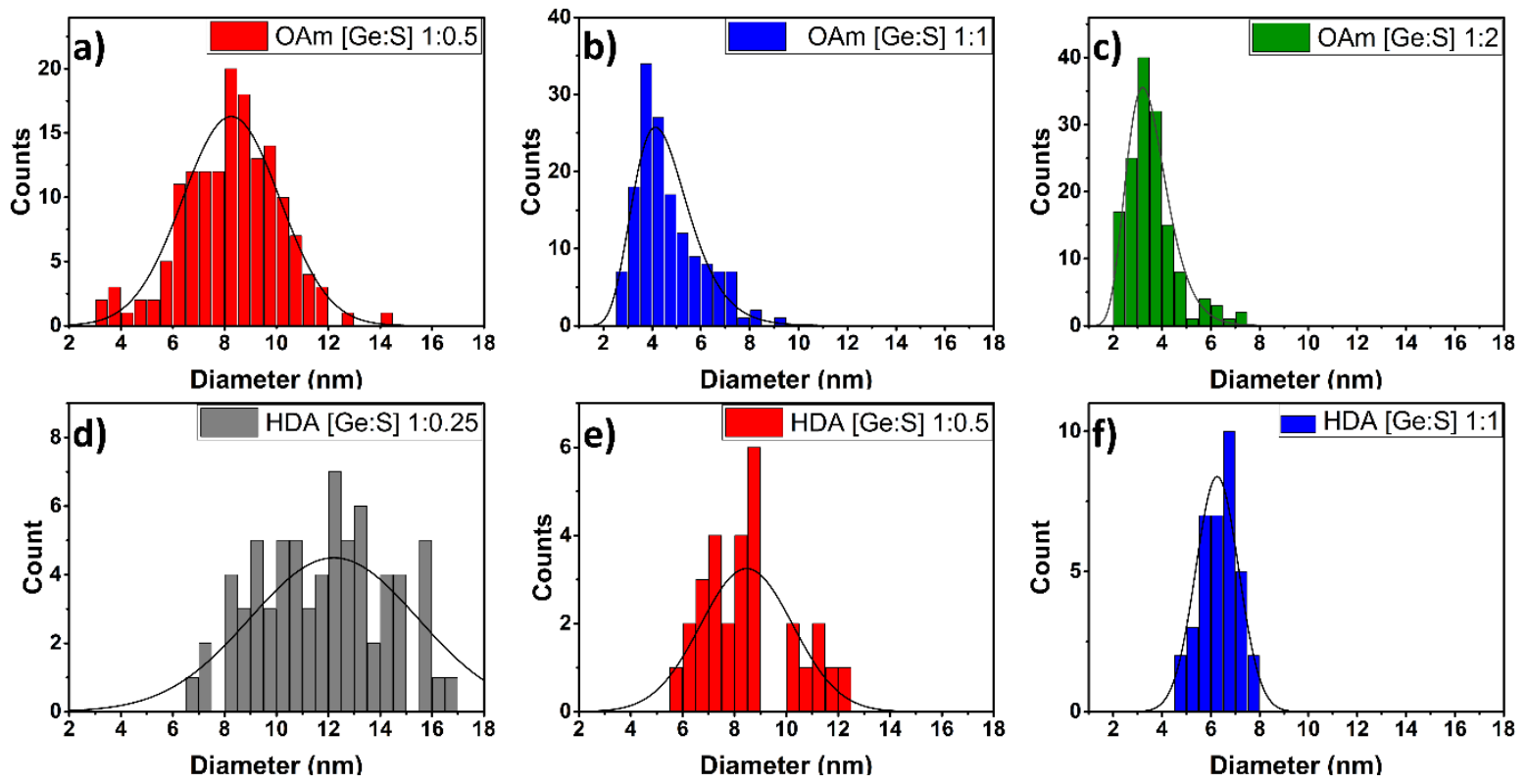

Figure S3: Size distributions for OAm [Ge:S] a) 1:0.5, b) 1:1, c) 1:2, and for HDA [Ge:S] d) 1:0.25, e) 1:0.5, and f) 1:1. For OAm the minimum number of particles measured was 150 , for HDA the minimum number of particles was 29. The normal curve superimposed on the histograms is a guide for the eye. 


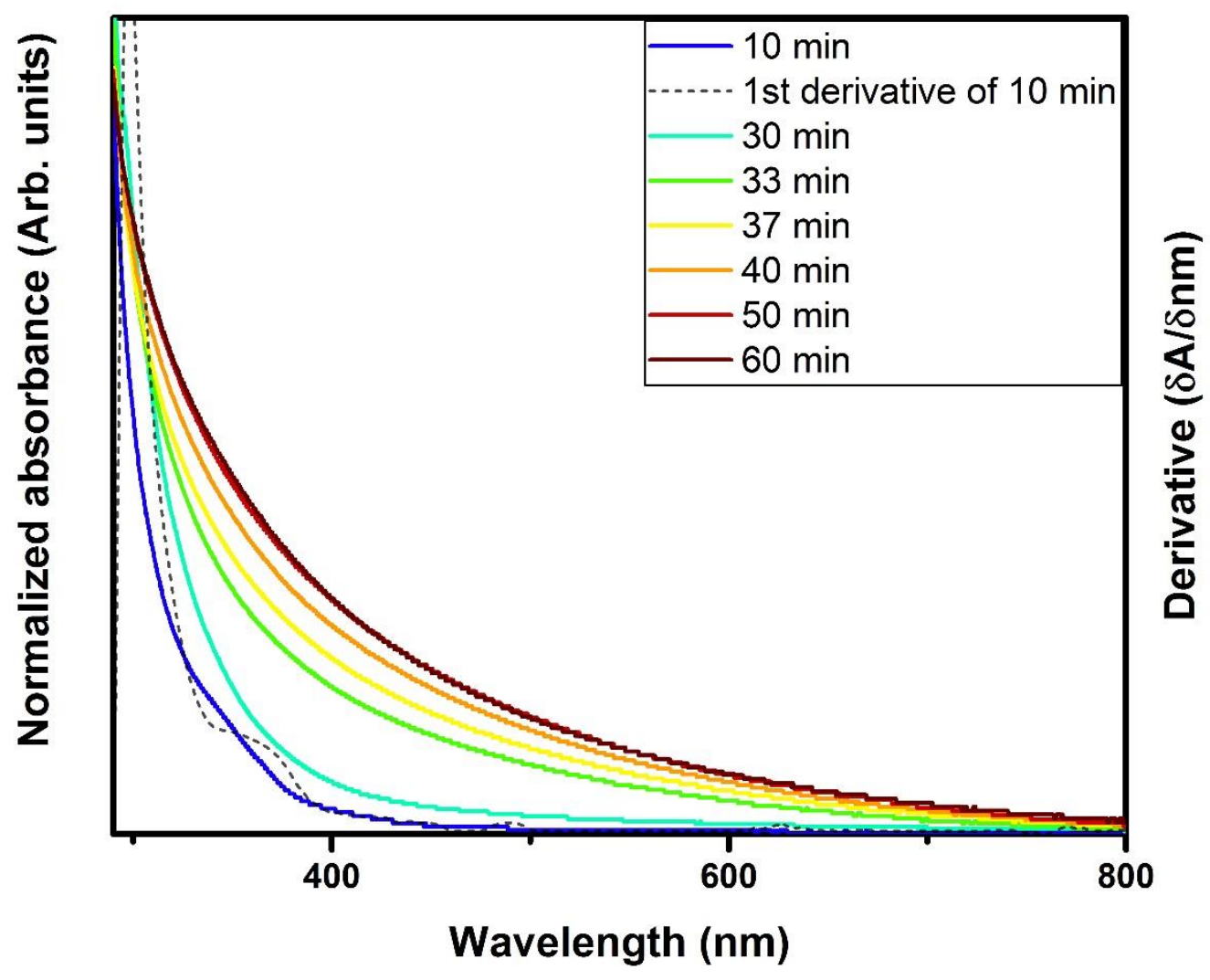

Figure S4: UV-Vis spectra of aliquots taken from a synthesis carried out in OAm with [Ge:S] 1:0.5. The derivative of the spectral line of the aliquot taken at $10 \mathrm{~min}$, is used to enhance the appearance of the spectral feature associated with nucleation. 


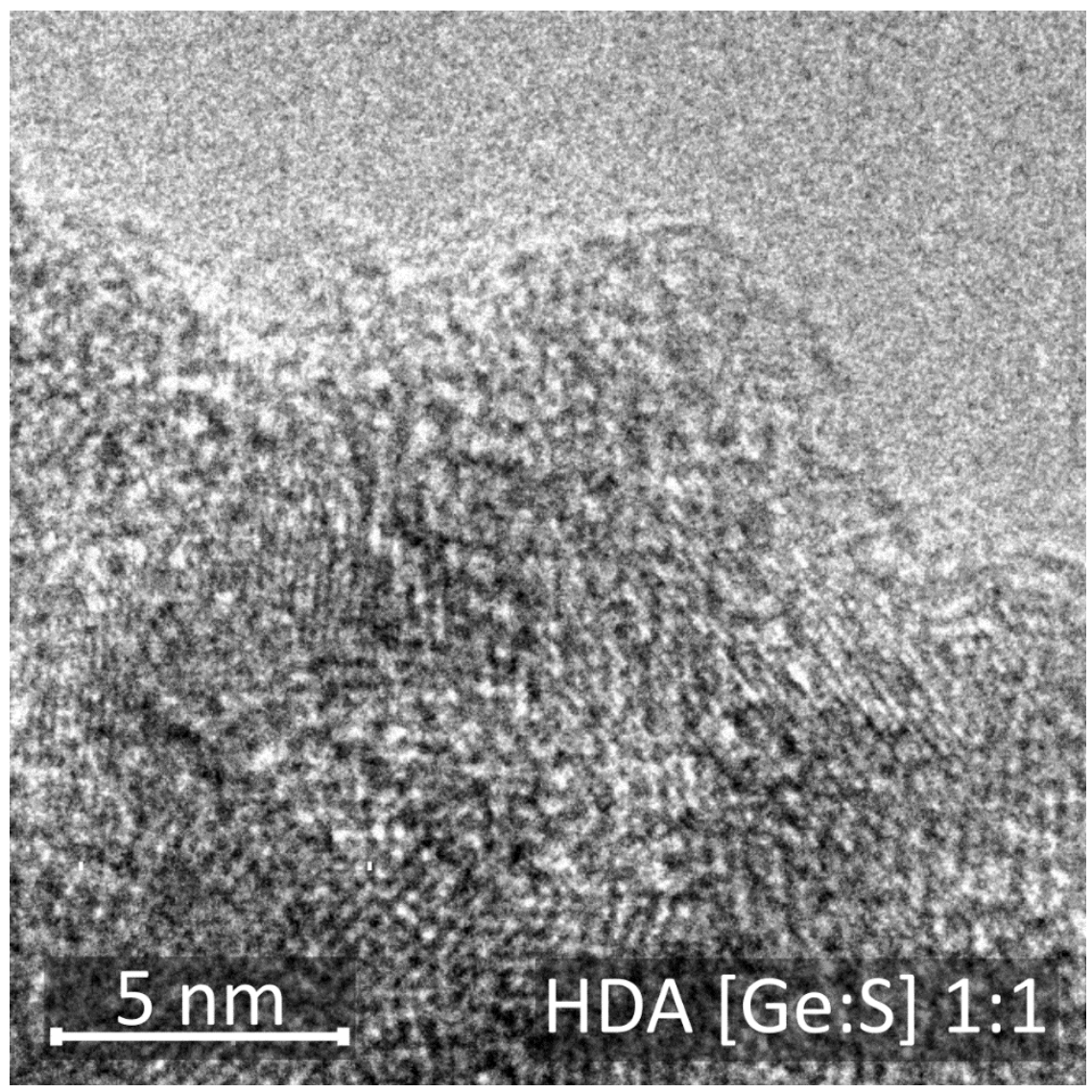

Figure S5: Scaled up portion of TEM image from an HDA [Ge:S] 1:1 synthesis showing the presence of crystalline lattice fringes. The picture shows the edge of a small NP cluster with multiple crystalline NPs. The images have been scaled up via post processing in the software imageJ. 


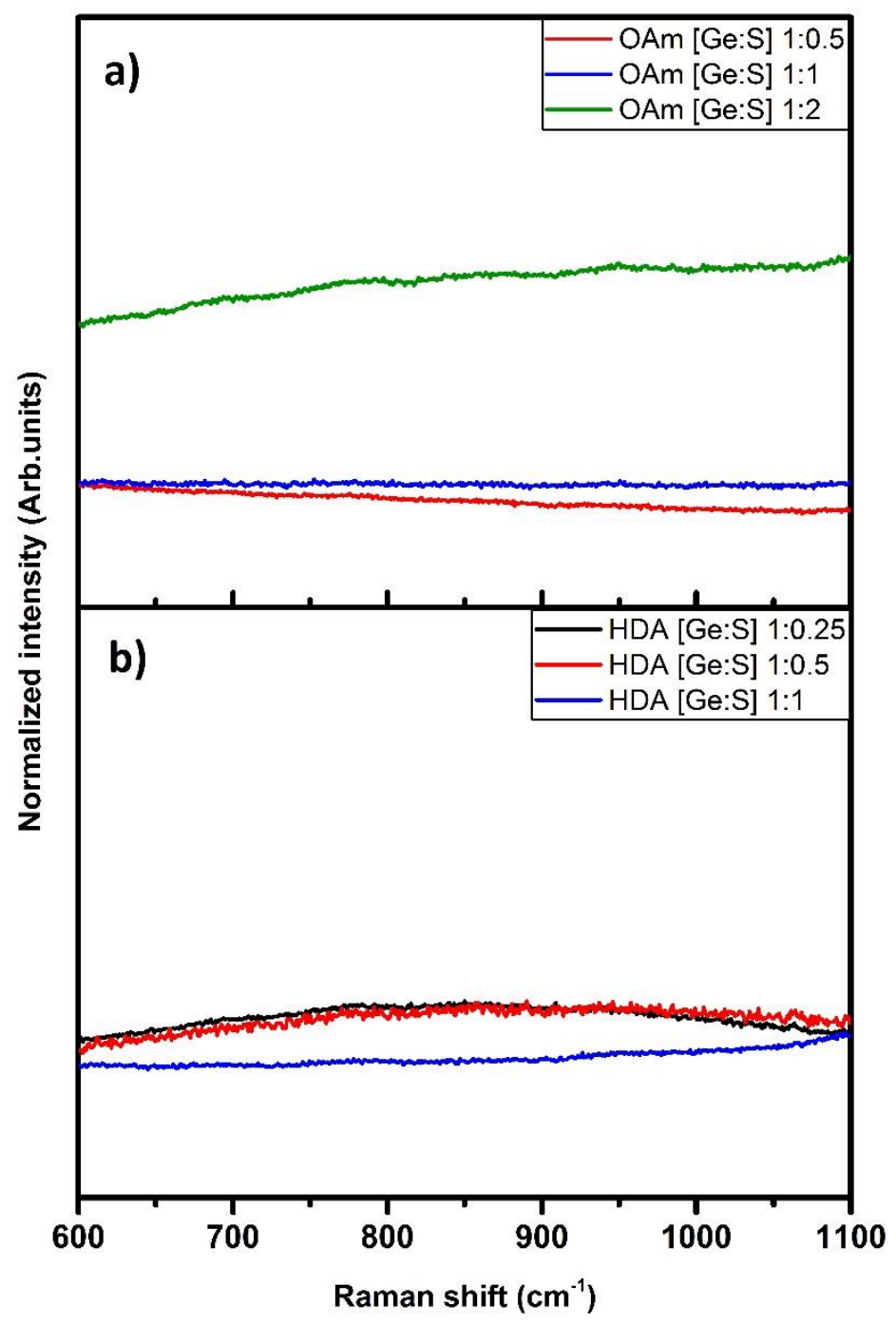

Figure S6: Raman spectra extended to higher Raman shifts from the normalized Raman spectra in Figure 2 of the main text for the samples produces in a) OAm and b) HDA, showing the absence of any features near $700 \mathrm{~cm}^{-1}$ and $1100 \mathrm{~cm}^{-1}$. This indicates a lack of covalent interaction between Ge and both $\mathrm{S}$ and $\mathrm{N}$, and confirming that no secondary species develop under our synthetic conditions. 


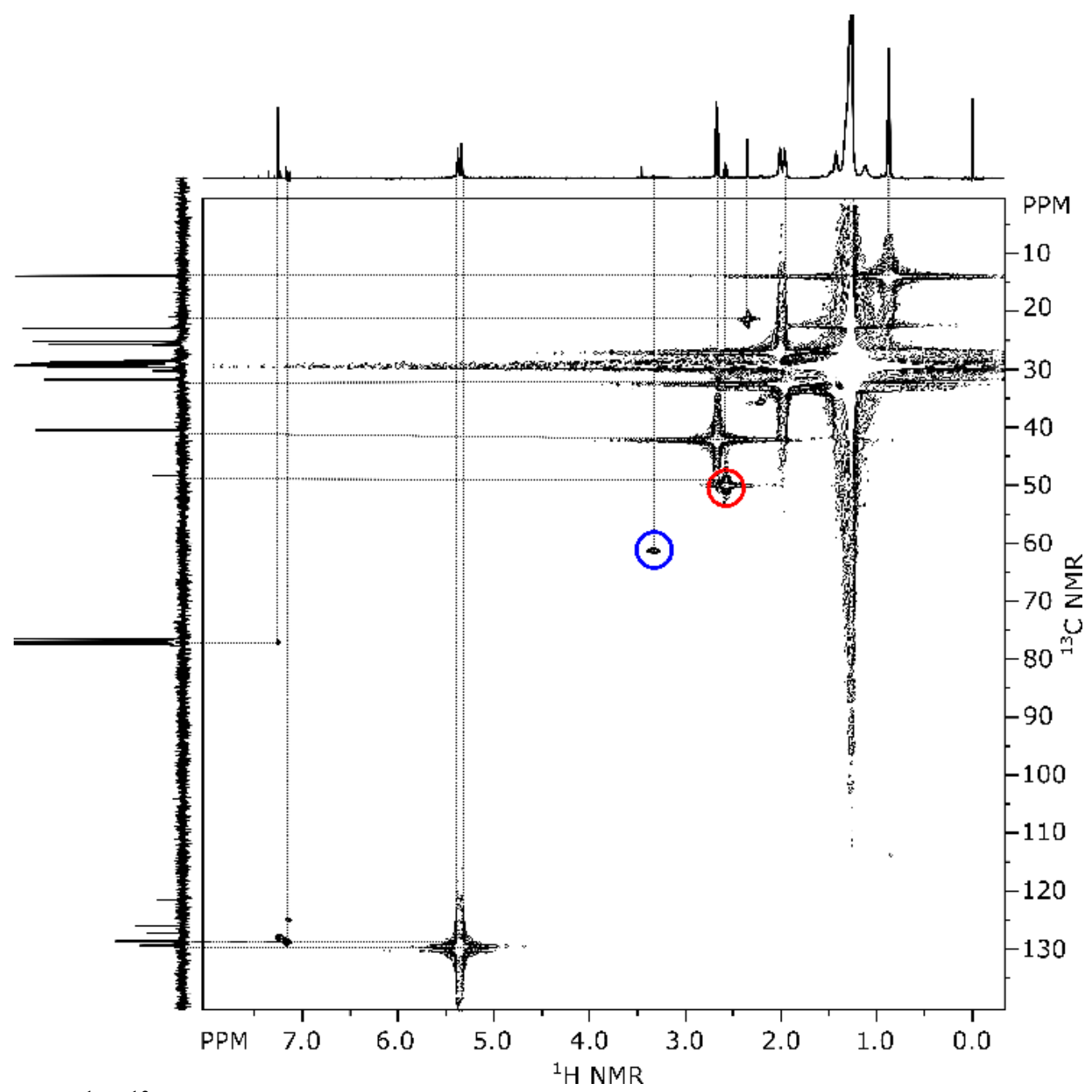

Figure S7: ${ }^{1} \mathrm{H}-{ }^{13} \mathrm{C}$ NMR HMQC spectrum of the reaction slurry prior to purification. The highlighted cross peak in the red circle shows a correlation of the proton feature located at $\delta 2.58$ ppm and the carbon peak at $\delta 47.5 \mathrm{ppm}$, which is coherent with a tertiary carbon $\alpha$ - to a C-S bond, as expected from the r-S-poly-OLA. The feature at $\delta 3.33 \mathrm{ppm}$ in the blue circle corresponds to the proton $\alpha$ - to a thioamide. The presence of a thioamide would result in a thioamidic carbon signal, which would be expected to evolve around $\delta 204 \mathrm{ppm}$ in the ${ }^{13} \mathrm{C}$ NMR, but which we have not observed. This is most likely due to the low intensity of quaternary carbons typically observed in NMR measurements, in tandem with a low concentration. 


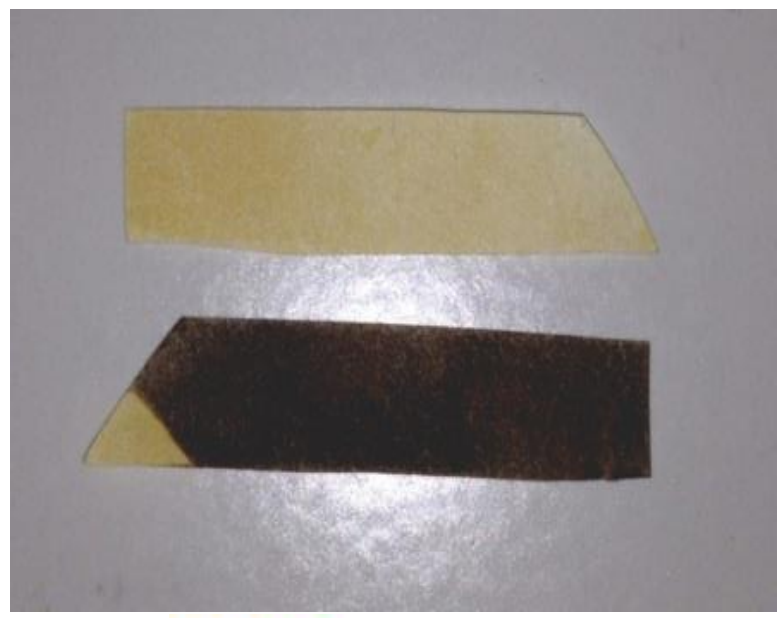

$\mathrm{PbO}+\mathrm{H}_{2} \mathrm{~S} \longrightarrow \mathrm{H}_{2} \mathrm{O}+\mathrm{PbS}$

Figure S8: photo of two pieces of $\mathrm{PbO}$ coated paper before (top) and after (bottom) exposure to the reaction atmosphere. The reaction scheme under the photo shows the production of $\mathrm{PbS}$ from $\mathrm{PbO}$ and $\mathrm{H}_{2} \mathrm{~S}$. 


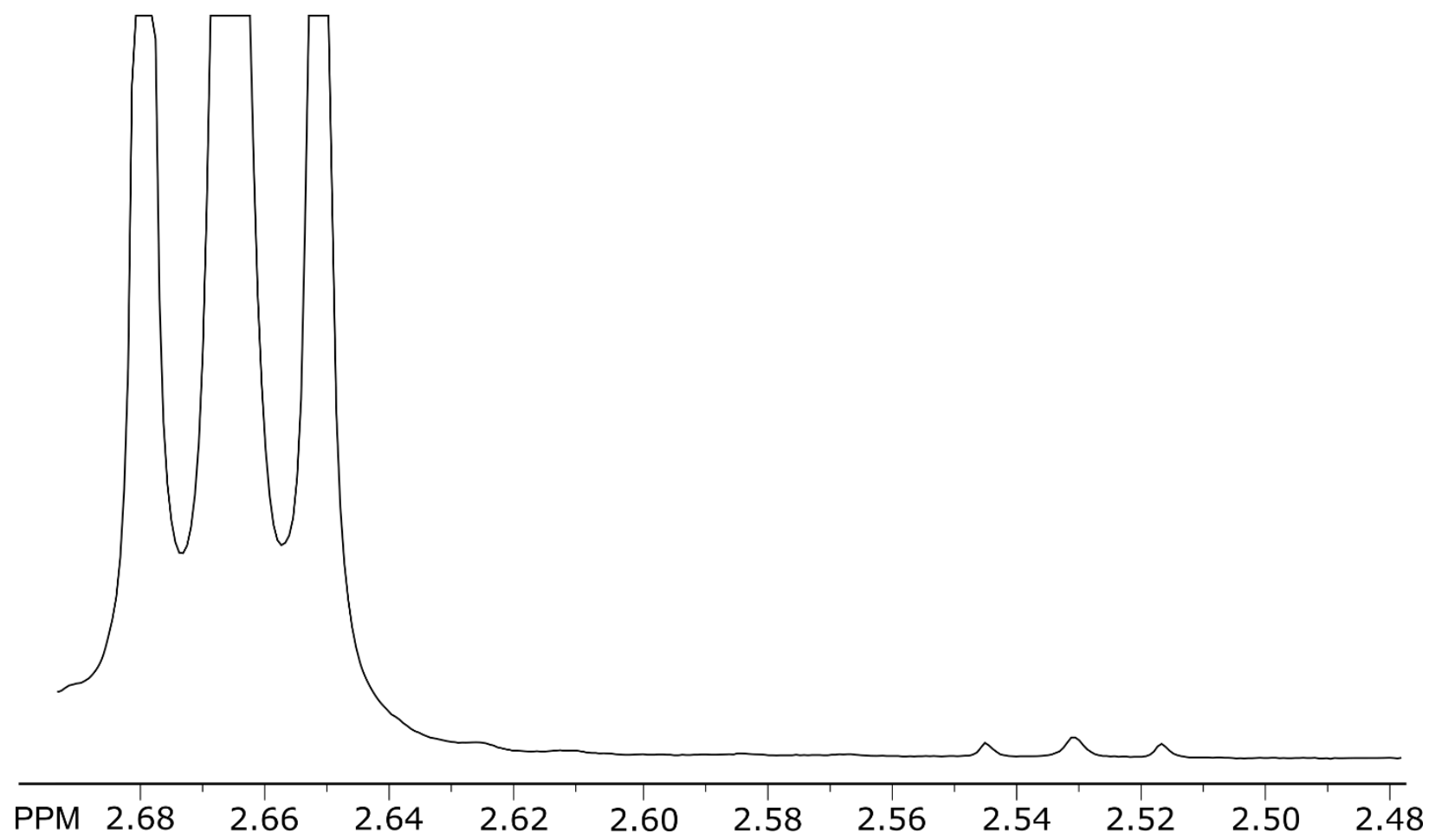

Figure S9: Scaled up section of the ${ }^{1} \mathrm{H}-\mathrm{NMR}$ spectra of the product of 2 (OAm after $\mathrm{S}$ addition) shown in Figure 4 in the manuscript. The small triplet at $\delta 2.53 \mathrm{ppm}$ is related to the production of the r-poly-OLA. Due to the lower temperature and shorter reaction time in respect to the crude, the intensity of the feature is extremely low. 